\title{
A case of myeloid sarcoma presenting with an orbital mass, hearing loss, and multiple cranial neuropathies
}

\author{
Seon-hee Lim, Hye-na Nam, Kyung-in Lim, In-sang Jeon \\ Department of Pediatrics, Gachon University, Gil Medical Center, Incheon, South Korea \\ E-mail: isjeon@gilhospital.com \\ Received: 5th June 2017, Accepted: 10th July 2017
}

\begin{abstract}
SUMMARY: Lim SH, Nam HN, Lim KI, Jeon IS. A case of myeloid sarcoma presenting with an orbital mass, hearing loss, and multiple cranial neuropathies. Turk J Pediatr 2018; 60: 322-325.

Primary myeloid sarcoma occurring in multiple sites; orbit, ear, brain, and spinal cord is a rare clinical entity. A 15-year-old male adolescent presented with bilateral orbital mass, hearing difficulty, and clinical signs of multiple cranial nerves palsy. Approximately 6 weeks later, acute myeloid leukemia was confirmed. This case alerts us that in patients with diverse sarcomatous lesions, acute myeloid leukemia presenting as myeloid sarcoma should be considered.
\end{abstract}

Key words: myeloid sarcoma, acute myeloid leukemia, $t(8 ; 21)$

Acute myeloid leukemia (AML), accounts for approximately $25 \%$ of childhood leukemia cases and is less common than acute lymphoblastic leukemia. ${ }^{1}$ AML can manifest as myeloid sarcoma (MS, chloroma, granulocytic sarcoma), an extra-medullary form of AML, or other myeloproliferative neoplasms. Although its incidence is only $3-8 \%$ of AML cases, MS is not rare in children diagnosed with an M2 FAB subtype AML. Moreover, it occurs more frequently in AML2 patients with $t(8 ; 21)$, that is, in up to $20-25 \%$ of cases. ${ }^{2}$ Furthermore, this translocation has been shown to generate a fusion gene known as RUNX1-RUNX1T1. ${ }^{3}$

The typical clinical features, such as, general constitutional symptoms and signs of bone marrow suppression, usually identify AML at diagnosis. However, the initial clinical manifestation of AML is occasionally nonspecific and creates diagnostic dilemmas. In particular, MS preceding AML may represent a diagnostic challenge. The clinical presentation of MS is extremely variable and depends on the organs involved. Although it can occur anywhere in the body, bone, periosteum, soft tissue, lymph nodes, and skin are the usual locations. ${ }^{4}$ Head and neck, including an orbit, are known locations, but extensive involvement of head and neck as in our case is not common, and the nonspecific symptoms presented by this extensive involvement, especially prior to a diagnosis of AML or the appearance of hematologic manifestations can delay diagnosis. ${ }^{5}$

We recently experienced an adolescent case of MS prior to the development of typical AML manifestations. Diagnosis was delayed because initial symptoms of eye discomfort and hearing difficulty led to his referral to an eye and ENT clinic. Here we report a case of a primary MS occurring in the multiple sites, that is, orbit, ear, brain, and spinal cord, which was diagnosed with AML with $t(8 ; 21) 6$ weeks later of the initial manifestation.

\section{Case Report}

A 15-year-old male adolescent visited our department of pediatrics with a chief complaint of an orbital mass which had occurred 4 weeks prior to this visit. Initially he visited a local ENT clinic for progressive right ear pain. Right orbital pain developed about 2 weeks later and he visited a local eye clinic. The patient did not have any particular symptoms for leukemia and his laboratory examinations, including complete blood cell count, were not suggestive of leukemia at that time. However, subsequently these symptoms aggravated and symptoms of cranial nerve palsy, including facial nerve palsy, 
developed. Therefore, he was referred to our hospital and physical examination revealed a confused mental state and right orbital swelling (Fig. 1A). Neurologic examination showed signs of cranial neuropathies (3-12th cranial nerves), including right eye lateral gaze limitation, medial deviation, right ptosis, dysarthria, bilateral facial palsy (peripheral type), right hearing loss, and paresthesia of both lower extremities. Splenomegaly, hepatomegaly, enlarged lymph node, and other hematologic symptoms were not seen. His height was $167 \mathrm{~cm}$ (25 50 percentile), weight $75 \mathrm{~kg}$ (75 90 percentile), and body mass index $26.9 \mathrm{~kg} / \mathrm{m}^{2}$. Complete blood count revealed; hemoglobin $5.0 \mathrm{~g} / \mathrm{dl}$, white blood cell count $25,580 / \mathrm{mm}^{3}$, reticulocyte $3.6 \%$, and platelet count $11,000 / \mathrm{mm}^{3}$. His differential leukocyte count revealed immature cells $81 \%$, promyelocytes $1 \%$, myelocytes $1 \%$, neutrophils $0 \%$, lymphocytes $6 \%$, eosinophils $1 \%$, and normoblasts $1 \%$. Biochemical analysis showed; total protein $7.7 \mathrm{~g} / \mathrm{dl}$, albumin $4.1 \mathrm{~g} / \mathrm{dl}$, AST $27 \mathrm{IU} / \mathrm{L}$, ALT $48 \mathrm{IU} / \mathrm{L}$, alkaline phosphatase $82 \mathrm{IU} / \mathrm{L}$, total bilirubin $0.6 \mathrm{mg} / \mathrm{dl}$, sodium 134 $\mathrm{mEq} / \mathrm{L}$, potassium $4.0 \mathrm{mEq} / \mathrm{L}$, calcium $8.5 \mathrm{mg} /$ $\mathrm{dl}$, phosphate $4.5 \mathrm{mg} / \mathrm{dl}$, uric acid $6.8 \mathrm{mg} / \mathrm{dl}$, and LDH $4812 \mathrm{IU} / \mathrm{L}$. Bone marrow aspiration and biopsy were followed and showed that about $81.4 \%$ of all marrow nucleated cells were blasts. Flow-cytometry showed the blasts were positive for CD45, CD34, MPO, CD117, $\mathrm{CD} 2$, and CD16. A subsequent cytogenetic and molecular study showed 46, X, -Y, +4, t $(8 ; 21)$ (q22;q22), 15pstak+; RUNX1-RUNX1T1. Brain MRI showed a mass in the superior and lateral aspects of the right orbit, and a smaller similar lesion in the superolateral aspect of the left orbit. Another enhancing lesion in the left high frontal scalp, otomastoid inflammation on both sides, and a T2 hyperintense lesion in the splenium and posterior body of the corpus callosum were also visualized (Fig. 2). L-spine MRI showed diffuse low signal intensity of lumbar and sacral bone marrow with diffuse enhancement.

According to the four phases of the CCG 2961 (induction, consolidation, intensification, and standard follow-up care), chemotherapy was immediately started. After induction therapy, clinically symptoms and orbital masses diminished and follow-up brain MRI revealed a reduction in the right orbital mass, and absence of the left orbit mass and of left high frontal scalp (Fig 1B). In addition, bone marrow was in a state of complete remission. Consolidation and intensification were performed using high dose Ara-c+idarubicin without any adverse event, except bone marrow suppression. Two and half years after completion of treatment, the mass-related symptoms and neuropathy were almost completely recovered, and bone marrow remained in a state of complete remission. Informed consent of the patient and his family were obtained.

\section{Discussion}

The mechanism responsible for the extramedullary proliferation of blasts of one or more myeloid lineages in MS has not been fully elucidated. Most often, MS is encountered concurrently in a patient with previously or recently recognized AML, and its presence predicts relapse in a patient thought to be in remission from previously diagnosed AML. ${ }^{6}$ Rarely, MS is identified in myelodysplastic syndrome or as an acute blastic transformation of myeloproliferative neoplasms. However, MS may precede the appearance of blood or bone marrow disease and when it does so, it is difficult to diagnose. Cases that present for de novo MS months or even years before the manifestation of AML have been reported as many as $27 \%$ of MS cases. Time between isolated MS discovery and bone marrow involvement has been reported to be 10 months. ${ }^{7}$ MS can affect any body site, but involvements of soft tissue, bone, skin, lymph node, or gastrointestinal tract are more common. Because locations are diverse, the clinical presentations of MS are also diverse as signs and symptoms are determined by tumor size and location. In our patient, the initial symptom was otalgia, which is not a common presentation. The differentiation of MS and otologic inflammatory disease is not straightforward. ${ }^{8}$ The orbital swelling present in our patient might have confused the diagnosis, as combined manifestations of the ears and eyes were easily confused with infectious diseases of the head. Initially, our patient was treated for an infectious disease until symptoms became aggravated. It is not clear whether hematologic abnormalities of AML were present when eye and ear symptoms occurred initially. However, his normal complete blood cell count and the 

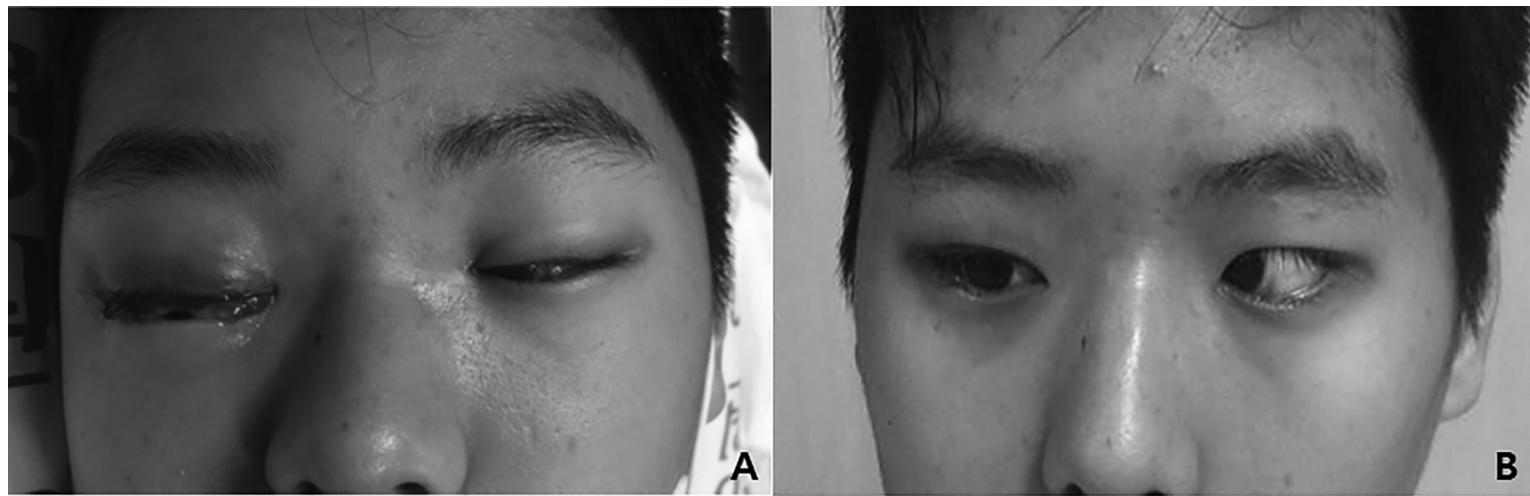

Fig. 1. A. Severe orbital swelling was noticed on the right side and slight swelling on the left side at initial visit to our hospital. B. Orbital swelling was much improved after induction chemotherapy.

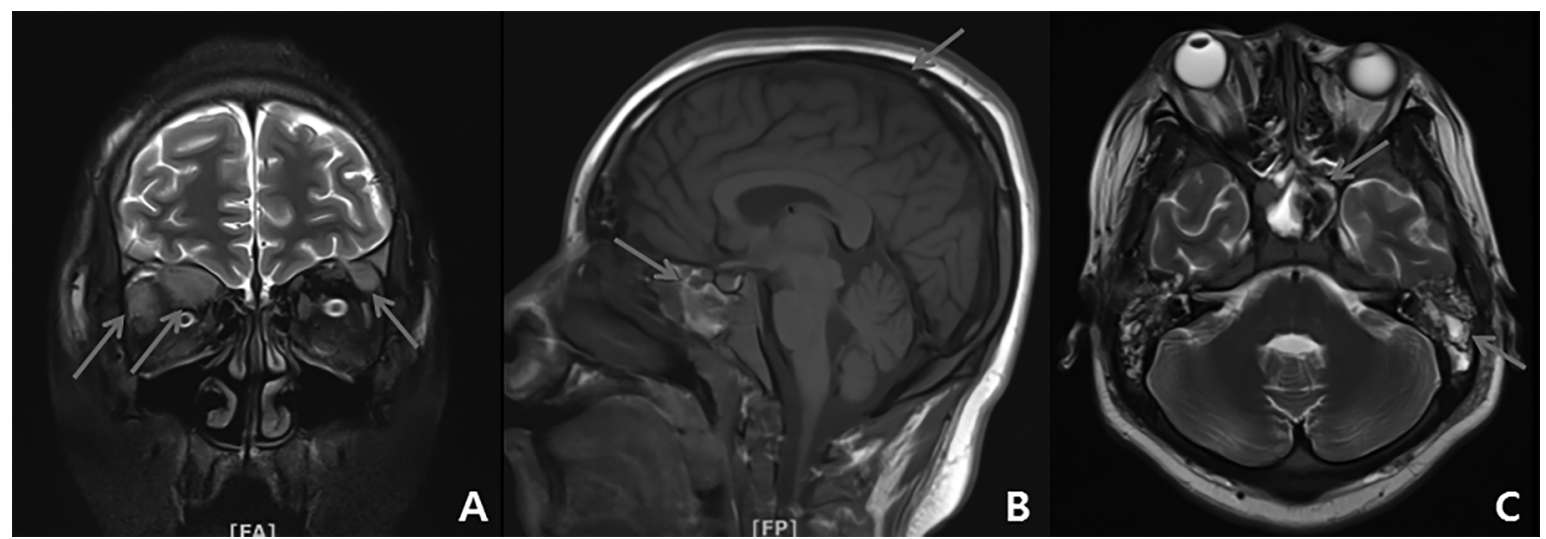

Fig. 2 A, B, C. Brain MRI imaging showed multifocal orbital masses at the extracornial spaces, low signal intensity lesions at the cranial bone marrow, and infiltrative lesions at the clivus. Additionally, a parenchymal lesion involving corpus callosum and inflammatory change at sphenoid \& mastoids were noticed

absence of clinical features of AML at that time suggest hematologic AML occurred near the time of its diagnosis.

Although suspicion of MS in the absence of a hematologic manifestation is difficult, clinicians should consider the possibility of MS when sarcomatous lesions are noticed. However, even when primary isolated MS is suspected and a mass biopsy is performed, pathologists find AML difficult to diagnose. In fact, the rate of misdiagnosis by pathology is as high as 25$47 \% .{ }^{9}$ Most of these cases have been mistaken for malignant lymphoproliferative disorders, such as, non-Hodgkin lymphoma, histiocytic lymphoma, thymoma, myeloma, eosinophilic sarcoma, extramedullary hematopoiesis, or mucosa-associated lymphoid tissue, and diagnoses were not corrected until AML was suspected by bone marrow aspiration and biopsy or peripheral blood smears. ${ }^{10,11}$ Pathologists recommend immunohistochemical staining with or without flow cytometry, cytogenetics, FISH, and molecular studies for the correct diagnosis of isolated MS, when morphological features raise suspicion of its presence. The diagnosis of de novo MS is equivalent to a diagnosis of AML, and MS is known to be consistently positive for CD117, CD43, myeloperoxidase, CD68, and CD34.5 Accordingly, the initial diagnosis of MS with classic morphologic and immunoreactivity findings should not be difficult.

MS can be encountered at any age both but is more frequently found in pediatric patients with FAB AML2 and $t(8 ; 21)$. Other chromosomal changes, such as, $\operatorname{inv}(16), \mathrm{t}(9 ; 11)$, and 11q23 abnormalities, have been reported to lesser degrees. ${ }^{12,13}$ Of note, MS with $t(8 ; 21)$ is associated with orbital manifestations in children. The oncogene rearrangement caused by $t(8 ; 21)$ results in the production of RUNX1RUNX1T1, which blocks differentiation and promotes blast cell self-renewal, and thus, causes massive accumulations of blasts in bone 
marrow. However, the involvement of RUNX1RUNX1T1 fusion in MS at the molecular level remains to be determined. Due to its rarity, the prognosis of MS is not well known but is believed to be poor. ${ }^{10}$ Some subgroup analyses of MS in children have shown those with MS in sites other than skin have better prognoses than children with AML only or children with MS involving skin (with or without other sites). In addition, those with MS in sites other than skin were younger, more often had the FAB AML2 subtype, and had a higher incidence of $\mathrm{t}(8 ; 21)$ that other children. ${ }^{2}$

There are no large prospective randomized studies which have document optimal treatment. In particular, in primary isolated MS without evidence of leukemia the optimal treatment has not been established. However, the majority of cases of isolated MS progress to AML, and generally systemic chemotherapy is recommended as for AML. ${ }^{10}$ In our case AML was concurrently diagnosed with MS, and systemic chemotherapy, that is, CCG 2961 containing Ara-c, anthracycline, and etoposide, was performed without any local treatment, such as, radiotherapy or surgery. Clinical symptoms of MS gradually improved after chemotherapy and remission was maintained 2 years after completion of therapy. The WHO classifies myeloid neoplasms with $\mathrm{t}(8 ; 21)$ RUNX1-RUNX1T1 AML as AML with recurrent genetic abnormalities, which has a good prognosis. ${ }^{14}$ Furthermore, the management of this condition is mainly based on chemotherapy without hematopoietic stem cell transplantation. Our experience suggests chemotherapy used in children with FAB AML2 with $\mathrm{t}(8 ; 21)$; RUNX1-RUNX1T1 would be effective in MS with $t(8 ; 21)$; RUNX1-RUNX1T1 as well.

\section{REFERENCES}

1. Xie Y, Davies SM, Xiang Y, Robison LL, Ross JA. Trends in leukemia incidence and survival in the United States (1973-1998). Cancer 2003; 97: 2229-2235.

2. Dusenbery KE, Howells WB, Arthur DC, et al. Extramedullary leukemia in children with newly diagnosed acute myeloid leukemia: A report from the Children's Cancer Group. J Pediatr Hematol Oncol 2003; 25: 760-768.

3. Peterson LF, Zhang DE. The 8;21 translocation in leukemogenesis. Oncogene 2004; 23: 4255-4262.
4. Campidelli C, Agostinelli C, Stitson R, Pileri SA. Myeloid sarcoma: Extramedullary manifestation of myeloid disorders. Am J Clin Pathol 2009; 132: 426437.

5. Seifert RP, Bulkeley III W, Zhang L, Menes M, Bui MM A practical approach to diagnose soft tissue myeloid sarcoma preceding or coinciding with acute myeloid leukemia. Ann Diagn Pathol 2014; 18: 253-260.

6. Neiman RS, Barcos M, Berard C, et al. Granulocytic sarcoma: A clinicopathologic study of 61 biopsied cases. Cancer 1981; 48: 1426-1437.

7. Klco JM, Welch JS, Nguyen TT, et al. State of the art in myeloid sarcoma. Int J Lab Hematol 2011; 33: 555-565.

8. Chang KH, Kim DK, Jun BC, Park YS. Temporal bone myeloid sarcoma. Clin Exp Otorhinolaryngol 2009; 2: 198-202.

9. Yamauchi K, Yasuda M. Comparison in treatments of nonleukemic granulocytic sarcoma: report of two cases and a review of 72 cases in the literature. Cancer 2002; 94: 1739-1746.

10. Yilmaz AF, Saydam G, Sahin F, Baran Y. Granulocytic sarcoma: a systematic review. Am J Blood Res 2013; 3: $265-270$

11. Antic D, Elezovic I, Milic N, et al. Is there a "gold" standard treatment for patients with isolated myeloid sarcoma? Biomed Pharmacother 2013; 67: 72-77.

12. Paydas S, Zorludemir S, Ergin M. Granulocytic sarcoma: 32 cases and review of the literature. Leuk Lymphoma 2006; 47: 2527-2541.

13. Park KU, Lee DS, Lee HS, Kim CJ, Cho HI. Granulocytic sarcoma in MLL-positive infant acute myelogenous leukemia: fluorescence in situ hybridization study of childhood acute myelogenous leukemia for detecting MLL rearrangement. Am J Pathol 2001; 159: 20112016.

14. Chang M, Raimondi SC, Ravindranath $\mathrm{Y}$, et al. Prognostic factors in children and adolescents with acute myeloid leukemia (excluding children with Down syndrome and acute promyelocytic leukemia): univariate and recursive partitioning analysis of patients treated on Pediatric Oncology Group (POG) Study 8821. Leukemia 2000; 14: 1201-1207. 